\title{
Location measurement of an object using radio networks for Industry 4.0 applications
}

\author{
Alexander Fedotov", Vladimir Badenko, Vladimir Kuptsov, Sergei Ivanov and Igor \\ Struchkov \\ Peter the Great St.Petersburg Polytechnic University, 29 Polytechnicheskaya str., Saint Petersburg \\ 195251, Russian Federation
}

\begin{abstract}
Indoor positioning methods using radio networks are investigated. Time Difference of Arrival (TDOA) method is studied deeply, and the main problems are revealed. Application of ultra-wide band (UWB) radio technology to TDOA method is discussed, and limitations to UWB receiver and transmitter are revealed. These results are of great importance for the organization of unmanned moving devices management in the paradigm of fully autonomous Fabric of the Future in Industry 4.0.
\end{abstract}

\section{Introduction}

Many modern applications in Industry 4.0 needs high precision indoor positioning based on remote sensing methods. These applications include for example, warehouse management and tracking of robotic vehicles inside manufacturing space $[1,2]$. In such situation one of the most popular methods uses measurements that are based on receiving an electromagnetic signal transmitted from a tracked object by several spatially distributed base stations [3]. Commonly used measurements are time delay for an active variant of the positioning system [4] and time differences for the passive environment [5].

The choice of method depends on many conditions. These are the required positioning accuracy, the rate of interrogation, the number of positioned objects, the possibility of their time synchronization, the presence and nature of the interference, etc. [6, 7]. From a practical point of view, the simplest methods should be preferred, but with increased requirements for accuracy and in unstable conditions, this may not be enough. In a complex, constantly changing environment of large rooms, associated with the constant movement of a large number of people, the most suitable rangefinder parameters are obtained based on a two-sided assessment [8]. They do not require strict time synchronization, are more accurate, and require time synchronization only at base stations.

\footnotetext{
*Corresponding author: afedotov@spbstu.ru
} 


\section{Materials and Methods}

\subsection{Review and analysis of the field}

There are the following methods for determining the location and measuring the coordinates of an object in radio networks [9-11]:

- In RSSI (Received Strength Signal Indication) the distance to the object is estimated by the signal strength. The method is based on the assumption of a fairly rigid relationship between the degree of signal attenuation and the distance traveled. The main advantage of the method is simplicity. When using it, nothing is required except for fixing the received signal power from the positioned object at the base nodes. This method works well at short distances, but with an increase in the range, it gives a large error due to the specifics of the propagation of the radio signal;

- In AoA (Angle of Arrival) the location of the object is determined within the area of the triangle formed by the intersection of the axes of the antenna patterns of the sectors of three base stations (modified triangulation method);

- ToF (Time of Flight) is a measurement of the travel time of an electromagnetic wave from a mobile device (object) to an access point using a chirp signal;

- ToA (Time of Arrival) is a measurement of the signal travel time from the mobile terminal to the base station. The distance to the object is calculated based on the difference in the time of signal sending and receiving. Moreover, this method requires strict time synchronization on the sender and receiver, which is quite difficult to achieve. Synchronization error can significantly affect the ranging error;

- TDoA (Time Difference of Arrival) - the difference in the arrival time of a signal from a mobile device to several base stations is measured. Strict time synchronization is required only at base stations; no such requirements are imposed on a moving device;

- RTT (Round TripTime) - the base station sends a signal to a mobile device and waits for a response signal; the time difference between sending and receiving a signal is used to determine the travel time of the signal in both directions, and, consequently, the distance between objects;

- LPT (Location Patterning Techniques) - positioning is done using pattern recognition of radio signals, based on sampling and recording of radio patterns of signal behavior in a specific environment.

\subsection{TDOA positioning main problems}

In the TDoA method, the difference in the arrival times of the signal from the mobile device to the receivers is determined (Figure 1). This method requires synchronization of receivers, which can be achieved by using special measures such as the White Rabbit synchronization network $[12,13]$.

It is necessary to know the coordinates of at least three fixed receiving devices to calculate the coordinates of a mobile device on a plane using TDoA. Knowing the speed of the signal in the receiver and TDoA:

$$
\Delta t_{i j}=\frac{\left(D_{i}-D_{j}\right)}{c}, i, j=1,2,3
$$

we can determine the location coordinates of the moving device (2):

$$
D_{i}-D_{j}=\sqrt{\left(x_{i}-x_{M}\right)^{2}+\left(y_{i}-y_{M}\right)^{2}}-\sqrt{\left(x_{j}-x_{M}\right)^{2}+\left(y_{j}-y_{M}\right)^{2}}=\left(t_{i}-t_{j}\right) c
$$


where $t_{i}$ is time of arrival of the signal at $i$-th receiver.

The solutions of the equations in the plane $(x, y)$ will be three pairs of hyperbolas with foci at the locations of the receivers. Their intersection coincides with the location of the mobile device shown in Fig 1. The authors have developed a method using the exact solution of these equations [14].

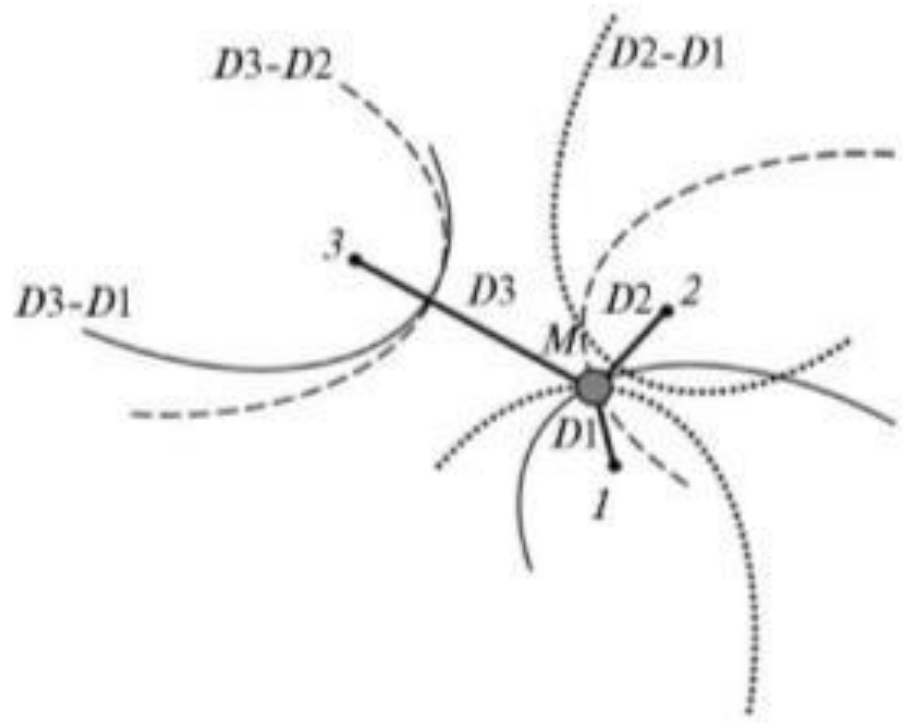

Fig 1. Positioning in the TDoA method, M - mobile device; 1, 2, 3 - receivers; D1, D2, D3 - the distance between the mobile device and the receivers; D2-D1, D3-D1, D3-D2 - hyperbolas obtained by solving equation (2)

Currently, systems are used to improve accuracy, in which various positioning technologies are combined [15-17].

Ultra-wideband (UWB) technologies provide the highest positioning accuracy. Ultrawideband (UWB) signals include signals with a central frequency $F_{c}$ and a band $\Delta F$, having a relative bandwidth $D=\Delta F / F_{c}>0.2 \ldots 0.25$, as well as signals with a band $\Delta F>500 \mathrm{MHz}$ (in the frequency range $3.1 \ldots 10.6 \mathrm{GHz}$ ). According to classical concepts, the magnitude of the error in determining the distance between the receiver and the transmitter or between the transceiver and the target when using the radar principle is inversely proportional to the frequency band, all other things being equal. Therefore, UWB systems are interesting for locating radiation sources. However, with a specific implementation of one or another distance measurement scheme, it is necessary to develop specific techniques and methods for realizing these potential capabilities.

The following UWB wireless technologies are currently in use [18]:

1. Ultrashort pulses. The pulse duration depends on the frequency range used, but is usually between 100 and 2000 ps. The property of these signals is the rigid connection of the pulse duration with the width of the power spectrum and its location on the frequency axis: the power spectrum extends from zero to the frequency $f \sim 1 / T$, where $T-$ is the length of the ultrashort pulse (Figure 2.a). Signal base $B \sim 1$.

2. Short radio pulses-oscillation trains. Within the framework of this approach, the signal is generated in a given frequency band. As in the case of ultrashort pulses, there is a strong relationship between the pulse duration and the signal power spectrum. The shape of 
the pulse envelope is chosen to be bell-shaped to obtain a more uniform spectral density in the frequency band (Figure 2.b). Signal base $B \sim 1$.

3. Chaotic radio pulses. The envelope of the power spectrum of these signals is determined by the initial spectrum of a continuous chaotic signal and, when certain conditions are met, practically does not depend on the pulse length (Figure 2.c). The signal base can vary widely.

4. Bursts of short pulses. As in the case of a single short pulse, the shape of the same pulses is consistent with the specified frequency band (Figure 2.d). The signal base is proportional to the number of pulses in the burst.

5. Signals with direct spread spectrum. This solution involves cutting a sinusoidal signal into very short fragments called "chips." A series of "chips" are used to transmit one bit. In the limit, when one "chip" is used to transmit one bit, this method coincides with the method of forming ultrashort pulses. The signal base is equal to the number of "chips" used to transmit one bit of information.
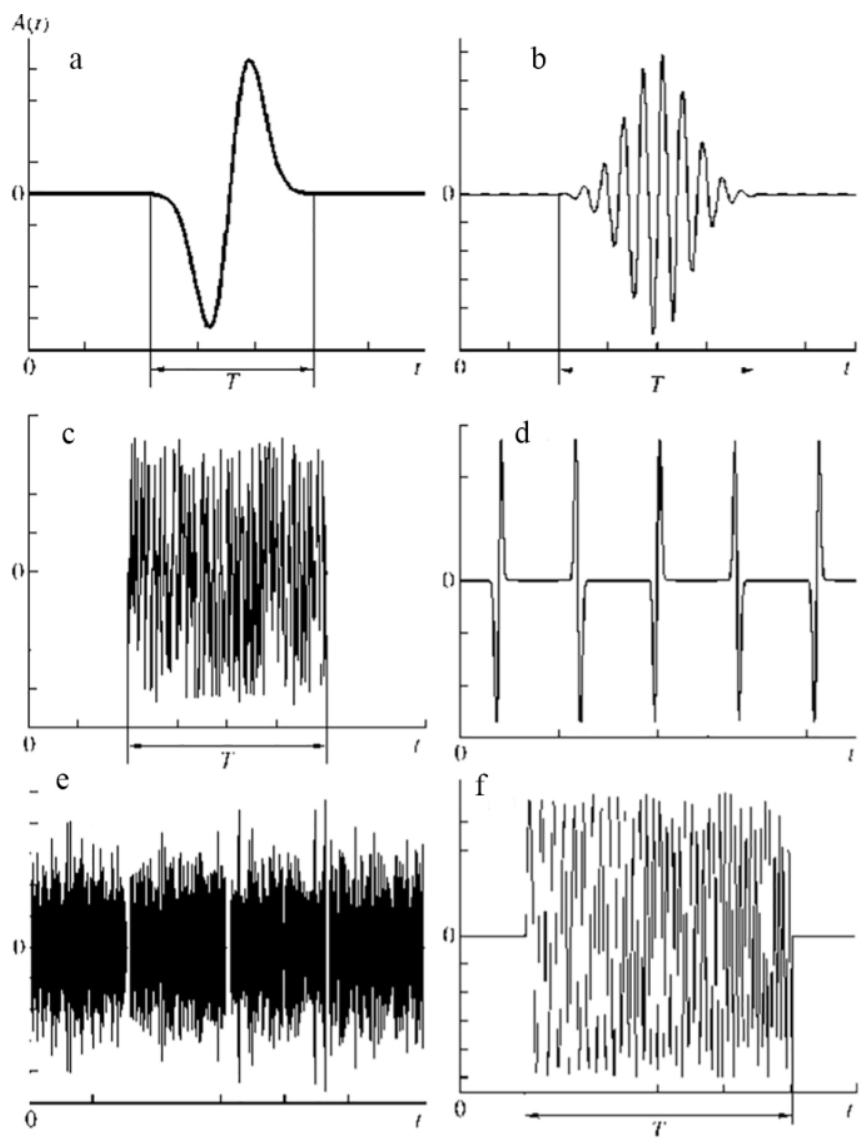

Fig 2. Fragments of UWB signals (dependence of amplitude $A$ on time $t$ ): a is an ultrashort pulse with duration $T ; \mathrm{b}$ is a short pulse formed from a fragment of a harmonic signal of duration $T$ with a Gaussian envelope; $\mathrm{c}$ is chaotic radio pulse of duration $T$; $\mathrm{d}$ is a pack of ultrashort pulses; e - signal with orthogonal frequency multiplexing; f is linear-frequency modulated pulse of duration $T$ 
6. Orthogonal frequency multiplexing (OFDM) signals. This type of signal has long been successfully used in radio communications (Figure 2.e). A feature of its use in UWB systems is a large spectrum width $(\sim 500 \mathrm{MHz})$ compared to OFDM signals used earlier.

7. Ultra-wideband signals based on frequency modulation (FM UWB). These signals are generated by scanning the frequency in the voltage controlled generators (Figure 2.f). With a single pass of frequency within a pulse, the base of the signal is proportional to the pulse length. The sweep rate determines the minimum pulse length at which full frequency sweep occurs. In this case, the signal base is equal to $B=\Delta T \Delta F$, where $\Delta T-$ is the pulse length; $\Delta F$ - tuning frequency band.

One of the problems of practical implementation of UWB systems is the synchronization of the transmitter and receiver. For example, in order to effectively implement coherent reception, it is necessary to ensure synchronization with an accuracy of no worse than $10 \mathrm{ps}$ for a pulse length of $150 \mathrm{ps}$. This is not an easy task, and technologies with significant energy consumption and rather complex circuitry are used to solve it [19]. The most preferred solution to synchronization problems is provided by the White Rabbit synchronization system, designed to synchronize the Hadron Collider devices at CERN. Currently White Rabbit has gone beyond the Hadron Collider project and is widely used in communication systems.

The moment of signal arrival can be determined by the leading edge of the envelope of the received radio pulse, as well as by the cross-correlation between the envelopes of the received signals at the outputs of analog-to-digital converters (ADC). The second method provides greater positioning accuracy $[14,20]$. The signal processing circuit in the receiving device for the second option is shown in Figure 3. The receiver consists of an antenna, a low-noise amplifier (LNA), a square-law detector, a low-pass filter (LPF) and an ADC.

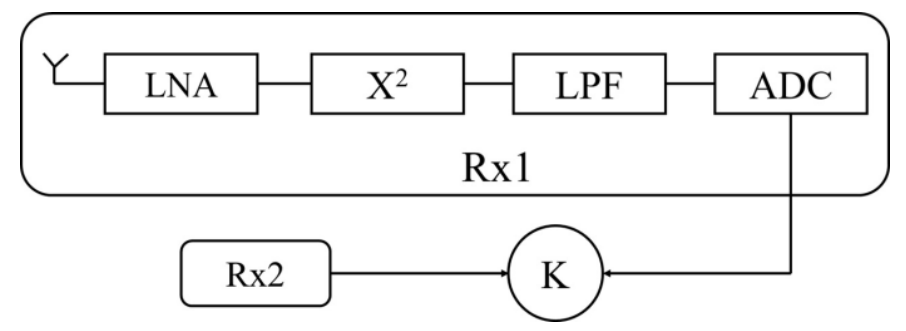

Fig 3. Scheme of signal processing in the receiving device: LNA - low-noise amplifier, X2 - squarelaw detector, LPF - low-pass filter, ADC - analog-to-digital converter, $\mathrm{K}$ - correlator that receives signals from receivers (Rx. 1 and Rx. 2)

In the considered scheme, to determine TDOA, signals at the ADC output are used, obtained as a result of squaring (the action of a square-law detector) of the received radio pulses and then passing through a low-pass filter with a cutoff bandwidth significantly lower than the bandwidth of the original radio pulses. All receivers are considered to be synchronized in time. Finding TDOA in two receivers is carried out by calculating the cross-correlation function of two signals received at the output of the receivers $y_{1}(t)$ and $y_{2}(t)$, and determining the moment in time when the maximum value of the crosscorrelation function (cross-correlation maximum) is observed [14]:

$$
k\left(y_{1}, y_{2}, \tau\right)=\int y_{1}(t) y_{2}(t-\tau) d t
$$




\section{Results and Discussion}

In the proposed method, the defining parameters are the length of the radio pulse $\Delta T$ and the low-pass filter bandwidth $\Delta f$. These parameters determine the base of the signal at the output of the receiver and set the error in determining the moment of arrival of the signal. For example, if the signal bandwidth at the LPF output practically coincides with the bandwidth of the original radio pulse, then the minimum value of the error in determining the signal arrival time corresponds to a value inversely proportional to $\Delta t=1 / \Delta F$, where $\Delta F$ - is the signal bandwidth. If the LPF band is matched to the length of the received radio pulse, then the position determination error will be approximately half the length of the radio pulse. Thus, TDOA measurement uncertainty can be expected to vary widely depending on the low-pass filter bandwidth of the receiver.

The wider the bandwidth, the more often the readings from the ADC output should follow. On the one hand, if you use a radio pulse bandwidth, for example, $2 \mathrm{GHz}$, then the counts should follow in $0.25 \mathrm{~ns}$. On the other hand, when the LPF bandwidth matches the $\mathrm{RF}$ pulse length, the sample rate will decrease. So, for example, with a pulse length of 100 $\mathrm{ns}$, time samples can follow at time intervals of $\sim 50 \mathrm{~ns}(20 \mathrm{MHz})$.

The first limiting case is difficult to implement but provides an error in determining the range of $\sim 7.5 \mathrm{~cm}$. The second limiting case is simpler from a technical point of view, but it has an expected error of $\sim 15 \mathrm{~m}$. Therefore, a reasonable compromise is required between these options. In addition, the technical limitations of the devices used must be taken into account. When developing receivers based on microcircuits, the LPF parameters can be set based on the desired TDOA determination error. In the considered scheme, with a fixed low-pass filter band, the signal's base (processing factor) as the output pulse of the envelope will be proportional to the length of the radio pulse. The increase in the noise immunity of the system and the effective use of all the energy of the received radio pulse is the result of the radio pulse duration increase. Such approach also distinguishes the proposed solution from determining the moment of arrival on the leading edge of the envelope of the received radio pulse.

\section{Conclusions}

1. The current indoor positioning situation includes the application of various positioning systems, inertial measurement units, and various assistive positioning technologies. The development trend of indoor positioning is aimed at high positioning accuracy for indoor applications in a wide range of applications.

2. The main directions of technology improvement include: seamless architecture of systems based on a heterogeneous network; key indoor technologies - decimeter accuracy; seamless GIS for indoor and outdoor spaces. The success of GNSS positioning in open space makes it possible to use them for closed spaces (for example, using pseudo-satellites).

3. This development of high precision technology for indoor positioning in a wide range of applications will provide a quantum leap in localization technology. High-precision seamless localization techniques for outdoor / indoor spaces will enrich people's lives and ensure public safety, for great economic and social benefits.

4. Unlike standard RFID and GPS systems, RTLS systems, built on the basis of ultrawideband transmitters, go beyond traditional technologies. The use of short pulses of energy moving at the speed of light makes it possible to obtain more accurate data on the movement of people and objects in space in real time.

The use of UWB technology for positioning systems has the advantage of providing high positioning accuracy over other technologies. Due to the short duration of one UWB pulse, signals are less susceptible to multipath and interference, and due to the high 
permeability of the ultra-wideband spectrum signal through the "electric slots" in obstacles, in different parts of the radio range, as well as the ability to work in conditions of high reflection, such signals propagate better in the presence of different kind of obstacles. Therefore, UWB technology does not require line-of-sight conditions between individual devices.

In addition, UWB signals have a sufficiently high noise immunity, which makes it possible to create more reliable positioning systems.

\section{Acknowledgments}

The work was financially supported by the Ministry of Science and Higher Education of the Russian Federation in the framework of the Federal Program "Research and Development in the Priority Areas of Development of the Russian Scientific and Technical Complex for 2014-2020", Agreement № 14.584.21.0035 Unique project identifier: RFMEFI58418X0035

\section{References}

1. Z. Zhao, J. Fang, G. Q. Huang, M. Zhang, iBeacon enabled indoor positioning for warehouse management, in 2016 4th international symposium on computational and business intelligence, ISCBI, pp. 21-26 (2016). DOI: 10.1109/ISCBI.2016.7743254

2. F. Bernardini, A. Buffi, D. Fontanelli, D. Macii, V. Magnago, M. Marracci, A. Motroni, P. Nepa, B. Tellini, Robot-based indoor positioning of UHF-RFID tags: The $S A R$ method with multiple trajectories. IEEE Transactions on Instrumentation and Measurement, 70, 1-15 (2021). DOI: 10.1109/TIM.2020.3033728

3. F. Zafari, A. Gkelias, K. K. Leung, A survey of indoor localization systems and technologies. IEEE Communications Surveys \& Tutorials, 21(3), 2568-2599 (2019). Doi: 10.1109/COMST.2019.2911558

4. G. Hu, P. Feldhaus, Y. Feng, S. Wang, J. Zheng, H. Duan, J. Gu, Accuracy improvement of indoor real-time location tracking algorithm for smart supermarket based on ultra-wideband. International Journal of Pattern Recognition and Artificial Intelligence, 33(12), 2058004 (2019). DOI: 10.1142/S0218001420580045

5. A. Motroni, A. Buffi, P. Nepa, A survey on Indoor Vehicle Localization through RFID Technology. IEEE Access, 9, 17921-17942 DOI: 10.1109/ACCESS.2021.3052316

6. M. Ficco, F. Palmieri, A. Castiglione, Hybrid indoor and outdoor location services for new generation mobile terminals. Personal and ubiquitous computing, 18(2), pp.271285 (2014). DOI 10.1007/s00779-013-0644-4P.

7. Z. Deng, Y. Yu, X. Yuan, N. Wan, L. Yang, Situation and development tendency of indoor positioning. China Communications, 10(3), pp.42-55 (2013). DOI: 10.1109/CC.2013.6488829

8. V.D. Kuptsov, S.I. Ivanov, A.A. Fedotov, V.L. Badenko Features of Multi-target Detection Algorithm for Automotive FMCW Radar. Lecture Notes in Computer Science, 11660. 355-364 (2019) DOI: 10.1007/978-3-030-30859-9_30

9. P. Pascacio, S. Casteleyn, J. Torres-Sospedra, E.S. Lohan, J. Nurmi, Collaborative Indoor Positioning Systems: A Systematic Review. Sensors, 21(3), p.1002 (2021). DOI: $10.3390 / \mathrm{s} 21031002$ 
10. F. Zafari, A. Gkelias, K. K. Leung, A survey of indoor localization systems and technologies. IEEE Communications Surveys \& Tutorials, 21(3), 2568-2599 (2019) DOI: 10.1109/COMST.2019.2911558

11. D. Dardari, P. Closas, P. M. Djurić, Indoor tracking: Theory, methods, and technologies. IEEE Transactions on Vehicular Technology, 64(4), pp.1263-1278 (2015). DOI: 10.1109/TVT.2015.2403868

12. J. E. Gilligan, E. M. Konitzer, E. Siman-Tov, J. W. Zobel, E. J. Adles, White Rabbit Time and Frequency Transfer Over Wireless Millimeter-Wave Carriers. IEEE transactions on ultrasonics, ferroelectrics, and frequency control, 67(9), 1946-1952 (2020) DOI: 10.1109/TUFFC.2020.2989667

13. A. Derviškadić, R. Razzaghi, Q. Walger, M. Paolone, The white rabbit time synchronization protocol for synchrophasor networks. IEEE Transactions on Smart Grid, 11(1), 726-738 (2020)

14. V. Kuptsov, V. Badenko, S. Ivanov, A. Fedotov, Method for Remote Determination of Object Coordinates in Space Based on Exact Analytical Solution of Hyperbolic Equations. Sensors, 20(19), 5472 (2020). DOI: 10.3390/s20195472

15. G. Deak, K. Curran, J. Condell, A survey of active and passive indoor localisation systems. Computer Communications, 35(16), 1939-1954 DOI: 10.1016/j.comcom.2012.06.004

16. B. Jang, H. Kim, Indoor positioning technologies without offline fingerprinting map: A survey. IEEE Communications Surveys \& Tutorials, 21(1), 508-525 (2019) DOI: 10.1109/COMST.2018.2867935

17. A. Nessa, B. Adhikari, F. Hussain, X. N. Fernando, A Survey of Machine Learning for Indoor Positioning. IEEE Access, 8, 214945-214965. (2020) DOI: 10.1109/ACCESS.2020.3039271

18. T. J. Chowdhury, C. Elkin, V. Devabhaktuni, D. B., Rawat, J. Oluoch, Advances on localization techniques for wireless sensor networks: A survey. Computer Networks, 110, 284-305. (2016) DOI: 10.1016/j.comnet.2016.10.006

19. A.S. Dmitriev, E.V. Efremova, A.V. Kletsov, L.V. Kuz'min, A.M. Laktyushkin, V.Y. Yurkin, Wireless ultrawideband communications and sensor networks. Journal of communications technology and electronics, 53(10), pp.1206-1216 (2008). DOI: $10.1134 / \mathrm{S} 1064226908100070$

20. S. Ivanov, V. Kuptsov, V. Badenko, A. Fedotov, An Elaborated Signal Model for Simultaneous Range and Vector Velocity Estimation in FMCW Radar, Sensors, 20, 5860 (2020) doi:10.3390/s20205860 